\title{
Extended Theories of Gravitation
}

\section{Structure of Spacetime and Fundamental Principles of Physics, following Ehlers-Pirani-Schild Framework}

\author{
Lorenzo Fatibene $^{1,2, a}$, Marco Ferraris ${ }^{1, b}$, Mauro Francaviglia ${ }^{1, c}$, and Guido Magnano, ${ }^{1,2, d}$ \\ ${ }^{1}$ Dipartimento di Matematica, University of Torino (Italy) \\ ${ }^{2}$ INFN - Sez. Torino, Iniziativa Specifica Na12
}

\begin{abstract}
Extended theories of gravitation are naturally singled out by an analysis inspired by the Ehelers-Pirani-Schild framework. In this framework the structure of spacetime is described by a Weyl geometry which is enforced by dynamics. Standard General Relativity is just one possible theory within the class of extended theories of gravitation. Also all Palatini $f(\mathcal{R})$ theories are shown to be extended theories of gravitation. This more general setting allows a more general interpretation scheme and more general possible couplings between gravity and matter. The definitions and constructions of extended theories will be reviewed. A general interpretation scheme will be considered for extended theories and some examples will be considered.
\end{abstract}

\section{Introduction}

General Relativity (GR) is a framework for what the standard structure of a meaningful physical theory should be, even before being a theory for gravitation meeting that standard. It defines how one can describe Nature in terms of observers, treating observers on equal footing, still providing an absolute description of spacetime Physics, i.e. one which is independent of the particular observer. Then it is an absolute description of gravity and observability of physical quantities in which spacetime is promoted to be a physical dynamical object. Finally, standard GR provides a particular dynamics for the gravitational field which rests on an action principle that was selected by Einstein and Hilbert essentially because of its simplicity. In standard GR physical and operational definitions of quantities such as, for example, distances and time lapses are provided too, related to theoretical quantities for which the theory can produce predictions which can be tested experimentally.

Since its definition back in 1916, standard GR has proven to be very effective in the description of gravity. It has proven to be better than Newtonian gravity in describing the Solar System. More than that, there is no difference in the Solar System tests between what is observed and what is predicted by standard GR. Recently a global positioning system (GPS) was set around the Earth which is using standard GR corrections to Newtonian physics. It is now clear that the GPS system simply would not

\footnotetext{
a e-mail: lorenzo.fatibene@unito.it

be-mail: marco.ferraris@unito.it

ce-mail: mauro.francaviglia@unito.it

de-mail: giudo.magnano@unito.it
} 
work without GR corrections and that it would be better to conceive a completely relativistic GPS which does not rest on Newtonian (wrong) assumptions.

Besides its success within the Solar System, standard GR has been used to describe the universe at galactic, extragalactic and cosmological scales. In order to describe (ordinary) matter one needs to make assumptions about particle Physics (part of which are tested within the Standard Model of Particle Physics (StMPP) and in colliders, see [1]).

The agreement between theory (theories, since this rests both on standard GR and StMPP) and observations is magnificent at astrophysical scales. Stellar models, neutron stars, binary systems are perfectly described by the theoretical models and observations fully confirm the theoretical predictions. The theory also predicted exotic astrophysical objects such as back holes which are now considered to be observed at astrophysical scales and most galaxies (and Milky Way is no exception) are believed to hide a galactic black hole in their center. We can even trace the orbits of stars around the black hole at the center of our galaxy.

Standard GR has been also used to study models for galaxies, clusters and the universe as a whole. At these scales the agreement between theoretical models and observations begins to be shaken from its foundations. The first hint came in fact from galaxies. Peripheral stars rotate around the galactic center with a period which is related (actually which defines) the gravitational mass of the galaxy. One can infer the (order of the) mass of the galaxy by measuring its luminosity (and making a number of educated guesses about the distance, the stellar population and about equivalence between inertial and gravitational masses) and find that the observed mass of the galaxy is less (sometime much less) than the gravitational mass expected by observing the star orbits; see [2]. This situation is usually considered as an evidence that some amount of matter is there without being detected in the luminosity budget. Once this dark halo is added the luminosity profile of a galaxy and its gravitational profile become essentially uncorrelated. Hence one can fit observations. If this can be satisfactory from the gravitational point of view it is considerably less well founded when it is considered in view of the StMPP. The dark sources are in fact dark since they escape the luminosity budget, but also because we do not know what they are made of. There is no candidate (except from neutrino components) for such matter sources in the StMPP. And the StMPP provides evidences according to which there should not be still-unknown candidates, unless one shakes its foundations quite severely. For those reasons those essentially unknown components are called dark matter.

Evidences of dark matter have been found at scales bigger than the galactic scale up to cosmological scales where the cosmic microwave radiation (CMB) keeps remembering the composition of the universe. At cosmological scales there are also evidences of other dark sources. They are responsible of the (positive) acceleration in the expansion of the universe. These dark sources cannot be ordinary matter since they must have negative pressure (sic!) to contribute positively to expansion of the scale factor. These dark sources are called dark energy and the best model for them is currently a tiny cosmological constant. Although the cosmological constant is difficult to be explained as vacuum energy in the StMPP, still it can be perfectly integrated with standard GR (and in fact it was).

Today there are many evidences for dark sources at different scales. At cosmological level one can estimate that about $(23.4 \pm 2) \%$ of the total mass in the universe is made of the so-called cold dark matter (CDM); see data from WMAP CMB of 2008, [3]. Then one has about $(72 \pm 2) \%$ of the so-called dark energy $(\Lambda \mathrm{DE})$ and about $(4.6 \pm 0.2) \%$ of ordinary baryonic matter. In other words the standard model for particle physics accounts for about only $4 \%$ of the total mass of the universe. The origin of the other $96 \%$ of the mass budget is still totally unknown. This is sometimes called the standard model for cosmology.

Of course, it may be considered frustrating to admit that one ignores the fundamental nature of most of the content of the universe still being so wonderfully able to predict its evolution. And one 
can speculate in many ways about explanations and models for dark sources. Higher dimensions and supersymmetry somehow naturally provide candidates for dark sources. However, there is no independent evidence for either of them. Particularly interesting is the Fermi survey; see [4]. This is supposed to detect (among other things) local gamma rays produced by annihilation of dark matter. To this point Fermi did not detect any signal which can be related to dark sources; it already ruled out many different models for dark matter and it has been almost reached the limit of noise under which nothing is expected to be detectable. Hence from many different directions one could say that there are strong evidences for dark sources, these evidences being however all from gravity physics while fundamental particle physics is not supporting in any known way their existence. Another possible way of reasoning is therefore to consider dark sources as purely gravitational phenomena. Instead of modifying Einstein equation of the right hand side one can try to coherently modify the dynamics of gravitational field itself (i.e. the left hand side of the equation) allowing mode general dynamics other than the simple dynamics of standard GR. Reasons to modify the gravitational theory from simple model of GR with the linear in the curvature Hilbert Lagrangian to more general theories with nonlinear Lagrangians come in fact also from other physical requirements dictated by recent issues like quantization and unification (á la string or mirror symmetries).

This has been shown to be possible. There are many models for modified gravity which account for the exotic behavior of gravitational physics without resorting to dark sources; see [2]. One can define more general dynamics than the Hilbert-Einstein action to describe gravity and which contain standard GR as a (usually somehow degenerate) representative. In these models the action depends on a generic function of curvature invariants, more general than simply the Ricci scalar. One refers to these models as Alternative Theories of Gravity (ATG), among which a rather promising class is that formed by so-called Extended Theories of Gravitation (ETG).

Of course in standard GR the interpretation of physical issues is well established and the relation between the theoretical representation of gravitational field is well related to observational protocols and operational definition of quantities such as length and time lapses. In standard GR there is a single metric which at the same time describes the gravitational field (both light cones and free fall) and provides a geometrical meaning for distances in spacetime. In ETG this situation is less well established; one has often more than one natural metrics to deal with and different aspects often refer to different metrics. Moreover, it is considerably less clear how to relate physical distances in spacetime to geometrical quantities, especially because the geometry in spacetime is more general and complex than in standard GR.

Luckily enough in the '70s Ehlers-Pirani-Schild (EPS) provided an axiomatic approach to the structures that a spacetime must have to fulfill the experimental needs and the fundamental principles (covariance, equivalence, causality) of gravitational physics which later showed to be suitable to provide a more general framework for interpreting gravity and spacetime measures in the wider context of ETG; see [5]. We shall give a more detailed account of EPS framework in Section 3. Now let us only notice that EPS framework does in fact provide many hints and insights about interpretation of ETG which must be considered before discussing whether ETG can be considered as physically sound theories for gravitation. In [6] we shall argue that, even if in the end standard GR will be finally confirmed as the only ETG which describes the correct physical gravitational field, still EPS and ETG will set it on a firmer ground by providing a more general interpretative framework in which one can test against observations some fundamental hypotheses that in standard GR are usually simply assumed to be true. 


\section{Higgs Particle and Equivalence Principle}

Recently the detection of the Higgs Boson has been announced in accordance with predictions of the StMPP. Of course data need to be confirmed and a better statistics should give indication on whether the particle detected is a fundamental scalar or not. Higgs field is known to be related to electroweak model and responsible for gauge Bosons $\left(W^{ \pm}, Z_{0}\right)$ to get masses by the symmetry breaking.

This is particularly interesting from the point of view of gravitational theories. In fact, Higgs Boson is an ingredient of the StMPP which, at least in the current understanding, has nothing to do with gravity. Of course, one can conjecture that the StMPP is an effective model of a wider still unknown model which takes gravity into account beside the standard interactions. This would probably be the approach of string theory or supergravity. It usually involves higher dimensions, superpartners and a lot of ingredients for which there is no observational evidence. Still it is a possibility to be considered.

On a more conservative viewpoint of GR, having inertial mass of particles decided within the StMPP is somehow puzzling. Firstly, inertial masses are related to gravitational masses by the (strong) equivalence principle which is considered an ingredient of our understanding of gravity. For this reason having a mechanism which messes up with inertial masses of particles in a model which seems to be independent of gravity is at least surprising. If the mechanism acts on some particles only, this is even more surprising since to the best of our understanding gravity is universal; see [7], [8].

Secondly, the StMPP is a model that fits with Special Relativity (SR). Special Relativity is however, a model for empty space, possibly with electromagnetic field, possibly with other massless particles, but it is fundamentally incompatible with masses as was already well known to Einstein, who was thence obliged to abandon flat models to describe gravity and masses in terms of curvature. Of course when one adds a mass to empty space in principle that generates a gravitational field, i.e. some form of curvature [9], which may be neglected but it is there. And the gravitational field is described by curvature of the geometric structure of spacetime (e.g. a Lorentzian metric or some gauge field) which has to be considered as dynamical and in the standard setting for GR cannot be simply Minkowski metric structure which is essential just for SR. Of course one can argue that for small masses this back reaction can be neglected for most practical purposes, which is certainly true. However, in principle the back reaction is there and it is enough to falsify flat SR in favor of curved GR. One can see the point by remarking that adding a point massive particle in a spacetime forces, in the simplest case, the metric to become the Schwarzschild metric. No matter how small the mass is, there are regions of spacetime in which the modification is dramatic and it is only keeping away from these regions that the mass can be neglected. In this solution an event horizon appears, a black hole is formed and spacetime has a non-removable singularity. Another way to see the same thing is is fact that, no matter how small the mass is there is a topological transition between Minkowski and Schwarzschild metric. Flatness is approximately preserved only at asymptotic level.

Thirdly, SR and GR are very different theories. In SR the metric is by definition a fixed and rigid structure of spacetime which, moreover, do not fit with GPS requirements that needs curvature to make it precise corrections. In GR (as well as in all ETG) it is instead dynamical. Even when one considers Minkowski metric as a possibly spacetime solution within GR that is quite a different theory with respect to SR. The general relativistic flat solution is a generally covariant model as any model of spacetime from GR, not only a Lorentz covariant model as in SR. This incommensurability among different models has longly been noticed; see [10].

Under many viewpoints, masses cannot be in principle neglected no matter how small and they are incompatible with SR framework. One can use SR as a computational tool, but the framework which has been and has to be really used is GR or any other coherent theory of curved spacetime. As a matter of fact particle physics is developed just within SR and it is widely known that most of its main concepts are not easily extendable to GR. Our current understanding of particles, quantum 
mechanics, perturbative methods, and many others are not friendly at all to our GR understanding. Higgs mechanism of generation of masses is just one of these situations in which the fundamental incongruence between SR and GR is highlighted.

Moreover, it is commonly believed that the weak equivalence principle lies at the bases of any relativistic theory and is a prerequisite for the possibility of geometrization of gravity, while the strong equivalence principle pinpoints standard GR among a wide class of possible relativistic theories of gravitation. Higgs mechanism, even in view of an unknown connections with gravity, does violate strong equivalence principle in the form:

The outcome of any local experiment (gravitational or not) in a freely falling laboratory is independent of the velocity of the laboratory and its location in spacetime.

We shall view below explicit examples of ETG in which gravitational interaction does depend on time. In any event at high energy $\left(W^{ \pm}, Z_{0}\right)$ are massless and they acquire masses at low energy. Under this viewpoint a symmetry breaking is a local experiment output which depends on the location of the laboratory!

This fact is particularly interesting to argue the other way around i.e. by remarking that Higgs mechanism, violating strong equivalence principle, does in fact support more general dynamics for gravitational fields than the standard GR.

\section{Ehlers-Pirani-Schild Framework}

In the early 70s Ehlers, Pirani and Schild (EPS) proposed an axiomatic framework for relativistic theories in which they showed how one can derive the geometric structure of spacetime from potentially observable quantities, i.e. worldlines of particles and light rays; see [5]. Accordingly, in the EPS framework the geometry of spacetime is not assumed but derived from more fundamental objects.

By assuming two congruences of worldlines for particles $(\mathcal{P})$ and light rays $(\mathcal{L})$ on a spacetimes manifold $M$, one can define out of light rays (i.e. out of the electromagnetic field) a conformal class of metrics $\mathbb{C}=[g]$. Two metrics $\tilde{g}, g \in \operatorname{Lor}(M)$ are conformally equivalent iff there exists a positive scalar field $\varphi$ such that $\tilde{g}=\varphi(x) \cdot g$. Let us remark that the choice of a representative $\tilde{g} \in \mathbb{C}$ is conventional and in fact part of the specification of the observer; conformal transformations are gauge transformations. Then particles free fall is described by a projective class $\mathfrak{P}=[\tilde{\Gamma}]$ of connections. Two connections are projectively equivalent iff they share the same autoparallel trajectories. In fact the connection $\tilde{\Gamma}_{\beta \mu}^{\prime \alpha}=\tilde{\Gamma}_{\beta \mu}^{\alpha}+\delta_{(\beta}^{\alpha} V_{\mu)}$ defines the same geodesic trajectories as $\tilde{\Gamma}_{\beta \mu}^{\alpha}$ for any covector $V_{\mu}$. In this case we say that $\tilde{\Gamma}$ and $\tilde{\Gamma}^{\prime}$ are projectively equivalent. Accordingly, free fall corresponds to a projective class $\mathfrak{P}=[\tilde{\Gamma}]$; see $[11]$.

Finally, we need a compatibility condition between the conformal class $\mathfrak{C}$ associated to light cones and the projective class $\mathfrak{P}$ associated to free fall. This is due by the simple fact that we know that light rays (and hence light cones) feel the gravitational fields as mass particles. Noticing that $g$ lightlike $g$-geodesics are conformally invariant (unlike general $g$-geodesics), we have then to assume that $g$-lightlike $g$-geodesics are a proper subset of all $\tilde{\Gamma}$-autoparallel trajectories. According to EPScompatibility condition one can show that a representative $\tilde{\Gamma} \in \mathfrak{P}$ of the projective structure can be always (and uniquely) chosen so that there exists a covector $A=A_{\mu} d x^{\mu}$ such that $\tilde{\nabla} g=2 A \otimes g$ where $g \in \mathfrak{C}$ is a representative of the conformal structure and the covariant derivative $\tilde{\nabla}$ is the one uniquely associated to $\tilde{\Gamma}$; see [12]. Equivalently one has

$$
\tilde{\Gamma}_{\beta \mu}^{\alpha}=\{g\}_{\beta \mu}^{\alpha}+\left(g^{\alpha \epsilon} g_{\beta \mu}-2 \delta_{(\beta}^{\alpha} \delta_{\mu)}^{\epsilon}\right) A_{\epsilon}
$$

To summarize, by assuming compatibility between particles and light rays one can define on spacetime a EPS structure, i.e. a triple $(M, \mathfrak{C}, \mathfrak{P})$. The conformal structure $\mathfrak{C}$ describes light cones and 
it is associated to light rays. Notice that having just a conformal structure one cannot yet define distances (which are not conformally invariant). Not being gauge covariant, in order to define distances one must resort to a convention which corresponds to the choice of a specific representative $g \in \mathfrak{C}$. On the other hand, the projective structure $\mathfrak{P}$ is associated to free fall so that one can make a canonical gauge fixing by choosing the only representative in the form (1) or, equivalently, the 1-form $A$.

The triple $(M, \mathfrak{C}, \tilde{\Gamma})$ (or, equivalently, the triple $(M, \mathfrak{C}, A))$ is called a Weyl geometry on spacetime. This setting is more general than the setting for standard GR where one has just a Lorentzian metric $g$ determining both the conformal structure $g \in \mathbb{C}$ and the free fall $\tilde{\Gamma}=\{g\}$ (i.e. the Levi-Civita connection uniquely associated to $g$ ). Hence standard GR is a simple and very peculiar case of EPS framework, where there is a gauge fixing of the conformal gauge. Such a fixing is possible iff the covector $A=A_{\mu} d x^{\mu}$ is exact, i.e. $A=d \varphi$. In this case, there exists a Lorentzian metric $\tilde{g} \in \mathbb{C}$ also determining free fall by $\tilde{\Gamma}=\{\tilde{g}\}$. When this happens the Weyl geometry $(M,[\tilde{g}],\{\tilde{g}\})$ is called an integrable Weyl geometry. Notice that this is still more general than standard GR in the sense that the metric determining free fall and light cones is not the original $g$ chosen to describe dynamics, but a conformally related one $\tilde{g} \in[g]$. Reverting to standard GR in a sense amounts to choose the gauge $\varphi$ to be a constant (so that $A$ vanishes identically).

In integrable Weyl geometries there is nothing ensuring that the canonical representative $\tilde{g}$ also gives us the measured distances, that as far as we can see could as well be related to any other conformally equivalent metric $g$. Fixing the metric that we use to calculate distances is, at the end, a choice that we can do only a posteriori, on the basis of observations, as Riemann clained as early as 1854 : the curvature of the universe has to be determined by astronomical observations.

At a fundamental level one can either decide to be strict on the interpretation of conformal gauge symmetry (and accordingly quantities that are not gauge invariant, such as distances, cannot be really observable) or one accepts conventional gauge fixing to define such quantities as observable, thus restricting symmetries of the system to the conformal transformations which preserve these gauge fixing. In the first case standard GR is equivalent to metric Weyl geometry (though we cannot measure distances) or, in the second case, we define distances but standard GR is not necessarily equivalent to metric Weyl geometries. Again, deciding which is the metric that really enters observational protocols is something that should not be imposed $a$ priori but rather something to be tested locally.

\section{Extended Theories of Gravitation}

In view of EPS analysis one should consider theories in which fundamental fields are a connection and a metric $(\tilde{\Gamma}, g)$, representing projective and conformal structures and a dynamics which implies the EPS-compatibility condition. Any such a model is called an extended theory of gravitation (ETG). If field equations also force the connection $\tilde{\Gamma}$ to be metric, then it is metric with respect to a conformal representative $\tilde{g}=\varphi \cdot g$, i.e. one has $\tilde{\Gamma}=\{\tilde{g}\}$. In this case the model is called an extended metric theory of gravitation.

Originally, Weyl proposed these structures to unify gravitational field and electromagnetism. This model was criticized, among other things, because in a generic Weyl geometry the length of rulers and time lapses, after parallel transport along a closed spacetime path, would depend on the holonomy of paths in spacetime. Of course in the original Weyl unified theory this effect is physically meaningful and the integrable case (with trivial holonomy) is not very relevant, since it corresponds to electrovac solutions. However, here the interpretation is quite different, totally gravitational, since the extra covector field $A$ should not be interpreted as the electromagnetic field, but rather as an additional degree of freedom for the gravitational field. As a consequence, it is physically reasonable to consider only extended metric theories of gravitation in which there is no holonomy effect affecting the length of rulers. 
ETG provide a wide class of models in which interpretation is quite firmly established by EPS and in which one can set experiments to decide which model is physically realized in our universe. Standard GR is only one possible model in this quite large family. It may be the real one, but, as we said, this should be discusses and decided against observations.

\subsection{Metric Affine formalism for $f(\mathcal{R})$ theories}

Let us here review Palatini $f(\mathcal{R})$-theories. They are extended metric theories of gravitation. Let $M$ be a spacetime manifold of dimension 4 endowed with a metric $g$ and a (torsionless) connection $\tilde{\Gamma}$ and let us consider a Lagrangian in the form

$$
L=\sqrt{g} f(\mathcal{R})+L_{m}(\psi, g)
$$

where $\mathcal{R}(g, \tilde{\Gamma}):=g^{\mu \nu} \tilde{R}_{\mu \nu}$, where $\tilde{R}_{\mu \nu}$ is the Ricci tensor of the independent connection $\tilde{\Gamma}$, where $f$ is a generic (analytic or sufficiently regular) function, and $\psi$ is a collection of matter fields.

With this choice we are implicitly assuming that matter fields $\psi$ is minimally coupled with the metric $g$ which in turn encodes the electromagnetic properties (photons and light cones). It would probably be better to be more liberal and allow matter couplings to the connection (see [13], [14], [15]). Let us here notice that what follows can be in fact extended to a more general framework; there are in fact matter Lagrangians depending on the connection $\tilde{\Gamma}$ in which field equations still imply the EPS-compatibility condition (1); see [13], [16], [17]. However, also in view of simplicity the matter Lagrangian $L_{m}$ is here assumed to depend only on matter and metric. Field equations of (2) are

$$
\left\{\begin{array}{l}
f^{\prime}(\mathcal{R}) \tilde{R}_{(\mu \nu)}-\frac{1}{2} f(\mathcal{R}) g_{\mu v}=\kappa T_{\mu \nu} \quad\left(T_{\mu \nu}=\frac{1}{\sqrt{g}} \frac{\delta L_{m}}{\delta g^{\mu \nu}}\right) \\
\tilde{\nabla}_{\alpha}\left(\sqrt{g} f^{\prime}(\mathcal{R}) g^{\beta \mu}\right)=0
\end{array}\right.
$$

where $f^{\prime}(\mathcal{R})$ denotes the derivative of the function $f(\mathcal{R})$ with respect to its argument $\mathcal{R}$. We do not write the matter field equations which will be considered as matter equations of state. The constant $\kappa=8 \pi G / c^{4}$ is the coupling constant between matter and gravity.

Under these simplifying assumptions the second field equations can be solved explicitly by introducing a conformal transformation $\tilde{g}_{\mu \nu}=f^{\prime}(\mathcal{R}) \cdot g_{\mu \nu}$. As a consequence the connection is given as $\tilde{\Gamma}_{\beta \mu}^{\alpha}=\{\tilde{g}\}_{\beta \mu}^{\alpha}$, i.e. the connection $\tilde{\Gamma}$ is the Levi-Civita connection of the conformal metric $\tilde{g}$. Thus in these theories the connection is a posteriori metric and the geometry of spacetime is described by an integrable Weyl geometry. The trace of the first field equation (with respect to $g^{\mu \nu}$ ) is so important in the analysis of these models that it has been called the master equation; [18]. It reads as

$$
f^{\prime}(\mathcal{R}) \mathcal{R}-2 f(\mathcal{R})=\kappa T:=\kappa g^{\mu \nu} T_{\mu \nu}
$$

where $T$ is the trace of the energy-mementum tensor $T_{\mu \nu}$. For a generic (sufficiently regular) function $f$, the master equation establishes an algebraic (i.e. not differential) relation which can be solved for $\mathcal{R}=r(T)$. The first field equation becomes then

$$
\tilde{G}_{\mu \nu}=\tilde{R}_{\mu \nu}-\frac{1}{2} \tilde{R} \tilde{g}_{\mu \nu}=\kappa\left(\frac{1}{\varphi(T)}\left(T_{\mu \nu}-\frac{1}{4} T g_{\mu \nu}\right)-\frac{1}{4} \hat{r}(T) g_{\mu \nu}\right)=: \kappa \tilde{T}_{\mu \nu}
$$

where we set $\varphi(T)=f^{\prime}(r(T))$. Accordingly we see that a Palatini $f(\mathcal{R})$-theory with matter behaves like standard GR for the conformal metric $\tilde{g}$ with a modified source stress tensor. Naively speaking, one can reasonably hope that the modifications dictated by the choice of the function $f$ can be chosen to fit observational data. 
In a sense, whenever $T \neq 0$ the presence of standard visible matter $\psi$, an energy momentum stress tensor $T_{\mu \nu}$ would produce by gravitational interaction with $\tilde{\Gamma}$ (i.e. with the conformal metric $\tilde{g}=\varphi(T) \cdot g$ ) a kind of effective energy-momentum stress tensor $\tilde{T}_{\mu \nu}$ in which standard matter $\psi$ is seen to exist together with dark (virtual) matter generated by the gauging of the rulers imposed by the $T$-dependent conformal transformations on $g$. In a sense, the dark side of Einstein equations can be mimicked by suitably choosing $f$ and $L_{m}$, as a curvature effect induced by $T=g^{\mu \nu} T_{\mu \nu} \neq 0$; see [19], [20], [21].

In vacuum or for purely electromagnetic matter obeying Maxwell equations, Palatini $f(\mathcal{R})$-theories are generically equivalent to Einstein models with cosmological constant (i.e. $\Lambda D E$ ) and the possible values of the cosmological constant form a discrete set which depends on the analytic function $f$. This is known as the universality theorem for Einstein equations (see [18]).

\subsection{Purely metric formalism for $f(\mathcal{R})$ theories}

In purely metric formalism one starts from a Lagrangian

$$
L=\sqrt{g} f(R)+L_{m}(\psi, g)
$$

where now the connection $\tilde{\Gamma}=\{g\}$ is assumed to be induced by the metric $g$ so that $\mathcal{R}$ reduces to the Ricci scalar $R$ of $g$. Field equations are now more complex since we vary only with respect to the metric $g$ which in turn induces a complex variation of the connection $\{g\}$. One eventually obtains

$$
\left\{\begin{array}{l}
f^{\prime}(R) R_{\mu \nu}-\frac{1}{2} f(R) g_{\mu \nu}-\nabla_{\mu} \nabla_{v} f^{\prime}(R)+g_{\mu \nu} \square f^{\prime}(R)=\kappa T_{\mu \nu} \\
f^{\prime}(R) R-2 f(R)+3 \square f^{\prime}(R)=\kappa T
\end{array}\right.
$$

where the second is the master equation obtained by tracing the first equation by $g$. In purely metric $f(R)$-theories the conformal factor $\varphi$ can be non-constant even for vacuum solutions and the space of solutions is richer than in the Palatini case. The dynamics for purely metric $f(R)$-theories can be recast by a Legendre transformation (see [22]) as

$$
L=\sqrt{g}(\varphi R+U(\varphi))+L_{m}(\psi, g)
$$

where we set $U(\varphi)=-\varphi \sigma(\varphi)+f(\sigma(\varphi))$ for the potential and the function $\sigma$ is obtained by the on-shell identity $\varphi=f^{\prime}(\mathcal{R})$ which can be solved as $\mathcal{R}=\sigma(\varphi)$. This is a Brans-Dicke theory with $\omega=0$, which can be recast as

$$
L^{* *}=\sqrt{\tilde{g}}\left[\tilde{R}-\frac{3}{2} \alpha^{2} \tilde{\nabla}_{\mu} \phi \tilde{\nabla}^{\mu} \phi+U\left(e^{\alpha \phi}\right)\right]+L_{m}^{*}(\phi, \tilde{g}, \psi)
$$

where we set $\varphi=e^{\frac{\sqrt{3}}{3}} \phi$; see [22]. One obtains standard GR (for the metric $\tilde{g}$ ) coupled with a standard scalar field $\phi$ with a potential dictated by $f$ and matter.

One could wonder whether there could be a relation between the scalar field $\phi$ and the Higgs boson of standard model of particles. Indeed, one may choose the potential to be $U(\phi)=b\left(\phi^{2}-a^{2}\right)^{2}$ which is the typical Higgs shape potential. This condition should be regarded, to this purpose, as a differential equation to single out an appropriate family of $f$ yielding the required potential for the scalar field.

However, there are also important differences with Higgs boson. First of all the Higgs boson in StMPP is a SU(2)-doublet and what has been detected is just its scalar remnant at low energies when the other component is used to break the electroweak symmetry.

Secondly, the Higgs boson has a preferred coupling to electroweak bosons $\left(W^{ \pm}, Z_{0}\right)$, while the conformal factor tends to have a universal coupling to all matter fields. Still with a suitable choice of the 
potential the mechanism to provide masses to particles can be at work and connected to gravitational physics.

Let us remark that there is no evidence yet that all details of Higgs physics correspond to what is expected within the StMPP nor that what has been detected is the only scalar field which can play the role of Higgs boson. On the contrary a field connected to the conformal factor could be the first observation linking StMPP and gravitational field, which if it were real could stay unnoticed for a relatively long time.

\section{Matter in Weyl Geometries}

In standard GR not any timelike congruence of trajectories is geodesic and one has strong constraints on possible motions of (perfect) fluid particles.

However, in the wider framework of Weyl geometries the metric structure is a gauge fixing of the conformal gauge freedom and the gravitational field is entirely encoded in the independent connection (or equivalently the covector $A$ ). Thus it is precisely Dirac theory of observability which indicates that one should be able to describing a fluid by any timelike congruences of curves. Then the gravitational field is described by the choice of a covector $A_{\epsilon}$ and this selects a class of possible fluid motions. Also the other way around; observing the motion of particles of a fluid one should be able to spot which gravitational field influences the fluid, making the gravitational field clearly observable through its effects on matter.

Let us hereafter briefly review the results of [23]. Let us consider, on a spacetime $M$, a conformal structure $(M, \mathfrak{C})$ and a $\mathfrak{C}$-timelike vector field $u$. The integral curves of $u$ are $\mathfrak{E}$-timelike trajectories by construction. For any gauge fixing of the conformal structure $g \in \mathfrak{C}$ one can normalize $u$ to be a $g$-unit vector. Let us denote the normalized vector by $n$.

Let $\gamma: \mathbb{R} \rightarrow M$ be an integral curve of the vector field $n$. We can arbitrarily reparametrize the curve $\gamma$ to obtain a different representative $\gamma \circ \phi$ of the same trajectory, the change of parameter being described by the diffeomorphism $\phi: \mathbb{R} \rightarrow \mathbb{R}$. If the original curve $\gamma$ was a $\Gamma$-geodesic motion (for a connection $\Gamma$ ) then $\gamma \circ \phi$ is a $\Gamma$-geodesic trajectory. Accordingly, one has

$$
n^{\mu} \nabla_{\mu}^{(\Gamma)} n^{\alpha}=\varphi \cdot n^{\alpha}
$$

for some scalar field $\varphi(x)$.

In standard GR one has no much choice for the fluid flow lines generated by $n$; the connection is freezed to be the Levi-Civita connection of $g$ and the vector field $n$ has to be selected in the small class of geodesic fields. In a Weyl setting one has a wider freedom in chosing the connection in the class of EPS-compatible connections given by (1). One can rely on this freedom to show that for any timelike vector field $n$ there exists one and only one EPS-compatible connection $\Gamma$ for which $n$ is $\Gamma$-geodesic, i.e. (10) holds true. One can easily check that $A$ has to fixed as $A_{v}=n^{\mu} \nabla_{\mu}^{(g)} n_{v}+\varphi n_{v}$. Notice how, once $u$ is given and a parametrization of curves is fixed by choosing the scalar field $\varphi$, one can determine the covector $A$ and thence the connection $\Gamma$.

\section{Conclusions}

We proposed ETG as a family of theories of gravitation which includes standard GR, which has natural interpretation in terms of EPS framework and which is potentially able to model dark sources as effective sources generated at fundamental level by modifications of gravitational interaction.

Gravitation is understood as curvature effect. While in standard GR curvature needs to be understood in terms of metric curvature, in ETG spacetime geometry is described by Weyl geometries 
which are made of a conformal structure and a connection. Unlike in standard GR the connection is not completely determined by the metric (and contains extra degrees of freedom encoded by the covector $A$ ). This setting is more friendly to represent matter, which in principle is expected to generate a gravitational field as some sort of curvature. IIn Weyl geometries the connection, in the sense discussed above, can generate curvature without one being forced to change the metric. As a consequence any timelike congruence can be regarded as describing a flow of massive particles and it defines the connection (and hence the gravitational field) which justifies the worldline of particles. We have also discussed how Higgs mechanism can be used as a hint of violation of strong equivalence principle and hence as an indirect support for non-standard dynamics for gravity. In [6] we shall discuss in detail observable quantities in ETG and how observational protocols affect the equivalence among different models; see also [24].

\section{Acknowledgements}

We acknowledge the contribution of INFN (Iniziativa Specifica NA12) the local research project Metodi Geometrici in Fisica Matematica e Applicazioni (2011) of Dipartimento di Matematica of University of Torino (Italy). This paper is also supported by INdAM-GNFM.

\section{References}

[1] A. Ferretti, in these Proceedings

[2] S. Capozziello, M. Francaviglia, Journal of General Relativity and Gravitation 40, 357-420 (2008)

[3] E. Komatsu et al., ApJS 280, 330 (2009); arXiv:0803.0547

[4] The Fermi-LAT Collaboration, arXiv:1003.0895

[5] J. Elhers, F.A. E. Pirani, A. Schild, The Geometry of free fall and light propagation in Studies in Relativity, Papers in honour of J. L. Synge 6384 (1972)

[6] L. Fatibene, M.Ferraris, M. Francaviglia, G. Magnano, in these Proceedings

[7] N. Dadhich, Physics News, 39, 20 (2009); arXiv:1003.2359v1

[8] L. Fatibene, M. Francaviglia, G. Magnano, Int. J. Geom. Meth. Mod. Phys. (in press); arXiv:1106.2221v2

[9] B. Riemann, On the Hypotheses that lie at the Foundations of Geometry, Göttingen 1854

[10] T.S. Kuhn, The Structure of Scientific Revolutions, (University of Chicago Press, Chicago, 1996)

[11] J.A.Schouten, Ricci-Calculus: An Introduction to Tensor Analysis and its Geometrical Applications, (Springer Verlag, Berlin, 1954)

[12] N. Dadhich, J.M. Pons, Gen Rel. Grav. (in press); arXiv:1010.0869v3 [gr-qc]

[13] M. Di Mauro, L. Fatibene, M.Ferraris, M.Francaviglia, Int. J. Geom. Methods Mod. Phys. 7, Issue:5 (2010); arXiv:gr-qc/0911.284

[14] G.J. Olmo, P. Singh, Journal of Cosmology and Astroparticle Physics 0901:030, (2009); arXiv:0806.2783

[15] L. Fatibene, M. Francaviglia, S. Mercadante, Int. J. Geom. Methods Mod. Phys. 7, 1185 (2010); arXiv:0911.2981

[16] T.P. Sotiriou, Class. Quant. Grav. 26152001 (2009); arXiv:gr-qc/0904.2774

[17] T.P. Sotiriou, S. Liberati, Annals Phys. 322, 935 (2007); arXiv:gr-qc/0604006

[18] A. Borowiec, M. Ferraris, M. Francaviglia, I. Volovich, Class. Quantum Grav. 15, 43 (1998)

[19] S. Capozziello, M. Francaviglia, Journal of General Relativity and Gravitation 40, 357 (2008) 
[20] S. Capozziello, M.F. De Laurentis, M. Francaviglia, S. Mercadante, Foundations of Physics 39 1161 (2009) arXiv:gr-qc/0805.3642v4

[21] S. Capozziello, M. De Laurentis, M. Francaviglia, S. Mercadante, in Proceedings of the Conference "Univers Invisibile", Paris June 29 - July 3, 2009 - to appear in 2012

[22] G. Magnano, L. M. Sokolowski, Phys.Rev. D 505039 (1994); arXiv:gr-qc/9312008

[23] L. Fatibene, M. Francaviglia, arXiv:1106.1961v1 (2011)

[24] C. Rovelli, Class. Quantum Grav. 8297 (1991) 
